\title{
Die Perikope von der Hochzeit zu Kana (Joh 2:1-11) im Kontext der Spätantike
}

\author{
Hans Förster ${ }^{1}$ \\ Vienna
}

\begin{abstract}
One focus of the interpretation of the Wedding at Cana (John 2:1-11) is usually the abundance of wine. The amount of water in the six large jars, which is changed into wine, seems to be enormous. However, the amount is traditionally not put into the context of the average consumption of wine in antiquity. Archaeological and papyrological sources suggest that - in the context of antiquity - the amount seems not to be extraordinarily large. Rather, the amount of wine seems to be sufficient just for a "common household." This is important for the interpretation of the entire passage: The "abundance of wine" is very often related to the hypothesis that this supposed abundance is to be seen in the context of the attempt to surpass the cult of Dionysos.
\end{abstract}

Keyword

Gospel of John; Wedding at Cana; Dionysos; Papyrology; Archaeology

\section{Einleitung}

Zahlreiche Fragen sind mit dem Zeichen der Verwandlung von Wasser in Wein anlässlich der Hochzeit zu Kana verbunden, ${ }^{2}$ es , ist bis heute so etwas wie ein $\sigma \eta \mu \varepsilon i ̂ \nu ~ \alpha ่ \nu \tau i \lambda \varepsilon \gamma o ́ \mu \varepsilon v o v$ geblieben, obwohl es—oder vielleicht gerade weil es-immer neu die Fachautoren angezogen und die Federn in

\footnotetext{
1) Der Beitrag entstand im Rahmen eines Forschungsprojektes des Fonds zur Förderung der wissenschaftlichen Forschung (Austrian Science Fund/FWF-Projekt P22017-G15/P24649-G15). 2) Vgl. auch nur A. Smitmans, Das Weinwunder von Kana: Die Auslegung von Jo 2,1-11 bei den Vätern und heute (BGBE 6; Tübingen: Mohr Siebeck, 1966) 6: „Die Exegese stellt zu Jo 2,1-11 vor allem drei Fragen: Wie versteht der Evangelist den Gang seiner Erzählung, insbesondere den Zusammenhang von 2, 3-5? Welcher religionsgeschichtliche Hintergrund ist zu beachten? Was ist der Sinn der Erzählung, ihre Botschaft?“
} 
Bewegung gesetzt hat.“3 Das „für Jesus recht untypische Luxuswunder“4 führt zu Schwierigkeiten bei der Deutung, da das Wunder scheinbar „Donar besser anstehen" ${ }^{5}$ würde als Jesus.

Während die Hypothese einer Zeichen-Quelle lange Zeit breite Zustimmung fand, ${ }^{6}$ wurde sie inzwischen grundsätzlich in Frage gestellt, ${ }^{7}$ sodass „heute eine deutliche Mehrheit der Exegeten die Existenz dieser ,SemeiaQuelle، “ bezweifelt. ${ }^{8}$ Das Weinwunder ${ }^{9}$ und der Ort Kana ${ }^{10}$ begegnen nur im Johannesevangelium. Innerhalb der Erzählung wird der Wechsel von einer Thematisierung der Menge zur Qualität als möglicher Bruch gedeutet.11

Das eigentliche Problem ist jedoch die Frage, was dieses Zeichen genau bedeutet. Mehrheitlich wird der Bericht von der Verwandlung von Wasser in Wein als Reaktion auf einen Dionysos-Kult gesehen. ${ }^{12}$ Es finden sich

3) Vgl. M. Hengel, „Der ,dionysische‘ Messias: Zur Auslegung des Weinwunders in Kana (Joh 2,1-11),“ in M. Hengel, Jesus und die Evangelien: Kleine Schriften V (hrsg. C.-J. Thornton; WUNT 211; Tübingen: Mohr Siebeck, 2007) 568-6oo, hier 568 (= M. Hengel, „The Interpretation of the Wine Miracle at Cana: John 2:1-11, " in The Glory of Christ in the New Testament: Studies in Christology. In Memory of George Bradford Caird [hrsg. L.D. Hurst und N.T. Wright; Oxford: Clarendon Press, 1987] 83-112).

4) S. Bergler, Von Kana in Galiläa nach Jerusalem: Literarkritik und Historie im vierten Evangelium (Münsteraner Judaistische Studien 24; Münster/Berlin: Lit-Verlag, 2009) 1: „Seit Beginn meines Theologiestudiums hat mich das für Jesus recht untypische Luxuswunder, im Rahmen einer Hochzeitsfeier Wasser in Wein zu verwandeln, gestört.“

5) So E. Hirsch, Das vierte Evangelium in seiner ursprünglichen Gestalt verdeutscht und erklärt (Tübingen: J.C.B. Mohr [Paul Siebeck], 1936) 123.

6) Vgl. z.B. R.T. Fortna, The Fourth Gospel and its Predecessor: From Narrative Source to Present Gospel (Edinburgh: T.\&T. Clark, 1989) 49.

7) Vgl. G. van Belle, The Signs Source in the Fourth Gospel: Historical Survey and Critical Evaluation of the Semeia Hypothesis (BEThL 116; Leuven: University Press, 1994) 376, dessen Fazit aus seinen Studien über die Semeia-Quelle lautet: „On the basis of these remarks, I am inclined to refuse the semeia hypothesis as a valid working hypothesis in the study of the Fourth Gospel.“

8) Siehe U. Schnelle, „Aus der Literatur zum Johannesevangelium 1994-2010. Erster Teil: Die Kommentare als Seismographen der Forschung,“ ThR 75 (2010) 265-303, hier 289.

9) Siehe hierzu auch R.F. Collins, „Cana (Jn. 2:1-12) — The first of his signs or the key to his signs?,"IThQ 47 (1980) 79-95, hier 79.

10) Vgl. K. Wengst, Das Johannesevangelium. 1. Teilband: Kapitel 1-10 (2. Aufl.; ThKNT 4/1; Stuttgart: Kohlhammer, 2004) 108.

11) Collins, „Cana,“ $81-82$.

12) So erst kürzlich W. Eisele, „Jesus und Dionysos: Göttliche Konkurrenz bei der Hochzeit zu Kana (Joh 2,1-11), "ZNW 100 (2009) 1-28; siehe auch W. Lütgehetmann, Die Hochzeit von Kana (Joh 2,1-11): Zu Ursprung und Deutung einer Wundererzählung im Rahmen johanneischer Redaktionsgeschichte (BU 20; Regensburg: Friedrich Pustet, 1990) 317-318. 
jedoch auch Stimmen, die dies als „höchst fragwürdige Idee“"13 ablehnen. Für die Deutung kann man an johanneische Symbolik anknüpfen, ${ }^{14}$ ganze Monographien wurden zu dieser Frage verfasst. ${ }^{15}$ Grundsätzlich kann diese Geschichte als anstößig empfunden werden, ${ }^{16}$ weil Jesus hier wie ein Magier aufzutreten scheint, ${ }^{17}$ das Wunder wird auch als „besonders massive(s) Wunder" ${ }^{18}$ bezeichnet. Weil dieses erste Zeichen im Johannesevangelium für das Verständnis des gesamten Evangeliums von Bedeutung ist, ${ }^{19}$ wird sogar das gesamte Evangelium von der Konkurrenz zu Dionysos gedeutet. $^{20}$

Im vorliegenden Beitrag ist es unmöglich, alle anstehenden Fragen erschöpfend zu behandeln, vielmehr geht es um eine Kontextualisierung des Berichts in der antiken Umwelt. Die Struktur des Beitrags ist von der Materie her vorgegeben. Zuerst sind sehr kurz die mit Dionysos verbundenen Weinwunder anzuführen. Danach ist der Stand der Diskussion bezüglich einzelner Elemente der Erzählung darzustellen. In einem weiteren Schritt sind dann die Voraussetzungen, auf denen die Diskussion aufbaut, auf dem Hintergrund der archäologischen und papyrologischen Evidenz einer kritischen Würdigung zu unterziehen. Die Konsequenzen daraus sind im Rahmen der Ergebnisse aufzuzeigen.

13) H. Thyen, Das Johannesevangelium (HNT 6; Tübingen: Mohr Siebeck, 2005) 151.

14) Vgl. F.J. Moloney, Belief in the Word. Reading the Fourth Gospel: John 1-4 (Minneapolis: Fortress Press, 1993) 79 .

15) Vgl. Lütgehetmann, Hochzeit von Kana, und H. Riedl, Zeichen und Herrlichkeit: Die christologische Relevanz der Semeiaquelle in den Kanawundern Joh 2,1-11 und Joh 4,46-54 (RSTh 51; Frankfurt u.a.: Peter Lang, 1997).

16) Hengel, „Der ,dionysische“ Messias,“ 571: „Die anstößige $\alpha$ đo die altkirchlichen Erzähler zu apologetischen Abgrenzungen zwang und die natürlich der ,aufgeklärte‘ moderne Leser besonders empfindet, verstärkt die Neigung, sie grundsätzlich symbolisch-allegorisch zu deuten [...].“

17) M. Welker, „Weinwunder-Weinstock—-lebendiges Wasser-Geist: Die anstößige Botschaft auf der Hochzeit zu Kana," in Gott im Wort_-Gott im Bild: Bilderlosigkeit als Bedingung des Monotheismus? (hrsg. A. Wagner, V. Hörner und G. Geisthardt; Neukirchen-Vluyn: Neukirchener, 2005) 201-205, hier 201: „[... Jesus erscheint hier als Magier, der etwa 600 Liter Wasser in Wein verwandelt!“

18) R. Schnackenburg, Das Johannesevangelium. I. Teil: Einleitung und Kommentar zu Kap. 1-4 (6. Aufl.; HThK 4/1; Freiburg: Herder, 1990) 310-311.

19) Vgl. J. Zumstein, „Die Bibel als literarisches Kunstwerk—gezeigt am Beispiel der Hochzeit zu Kana (Joh 2,1-11), " in Geist im Buchstaben? Neue Ansätze in der Exegese (hrsg. T. Söding; QD 225; Freiburg i. Br. u.a.: Herder, 2007) 68-82, hier 71.

20) So P. Wick, "Jesus gegen Dionysos? Ein Beitrag zur Kontextualisierung des Johannesevangeliums," Biblica 85 (2004) 179-198. 


\section{Das Wunder bei der Hochzeit zu Kana und der Dionysos-Kult}

Weinwunder sind in der Antike bezeugt und in besonderer Weise mit dem Dionysos-Kult verbunden. ${ }^{21}$ Dieser Gott hat nach antiker Auffassung den Menschen den Wein als Geschenk für erfahrene Gastfreundschaft gegeben.22

Man versucht, die Entstehung der Erzählung aus einer Konkurrenz zum Dionysos-Kult zu erklären. Tatsächlich gab es in Skythopolis, dem alttestamentlichen Beth Shean, das „etwas über $30 \mathrm{~km}$ von Kana entfernt liegt, “23 unzweifelhaft einen wichtigen Dionysos-Kult. ${ }^{24}$ Plutarch bringt Dionysos in besonderer Weise mit Jerusalem in Verbindung. ${ }^{25}$ Und noch im Trullanum (691/692) findet sich ein Verbot, während der Brumalia beim Ausdrücken der Weintrauben den Namen des Dionysos anzurufen. ${ }^{26}$ Deswegen kann ein Bezug zwischen dem Dionysos-Kult und dem Weinwunder zu Kana nicht mit der Begründung abgelehnt werden, dass die Thematik des Weins nur eines und möglicherweise nicht einmal mehr das wichtigste der mit Dionysos verbundenen Wunder sei. ${ }^{27}{ }_{\text {„Die mutmaßliche }}$ Entstehung der Kana-Erzählung in einer Konkurrenzsituation zwischen

21) Siehe auch nur I. Broer, „Noch einmal: Zur religionsgeschichtlichen ,Ableitung“ von Jo 2,1-11, “ SNTU.A 8 (1983) 103-123; I. Broer, „Das Weinwunder zu Kana: Joh 2,1-11 und die Weinwunder der Antike, "in Das Urchristentum in seiner literarischen Geschichte. FS Jürgen Becker (hrsg. U. Mell und U.B. Müller; BZNW 100; Berlin/New York: De Gruyter, 1999) 291-308.

22) Vgl. Silius Italicus, Punica VII.186-194.

23) Eisele, "Jesus und Dionysos," 10.

24) Eisele, "Jesus und Dionysos," 15 .

25) Vgl. F. Siegert, „Griechische Mythen im hellenistischen Judentum, "in Griechische Mythologie und frühes Christentum (hrsg. R. von Haehling; Darmstadt: Wissenschaftliche Buchgesellschaft, 2005) 132-152, hier 151 unter Verweis auf Plutarch, Quaestiones convivales 4.6.

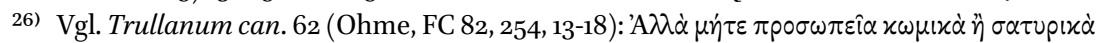

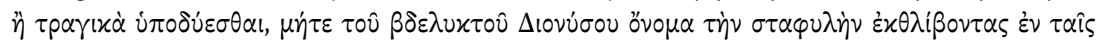

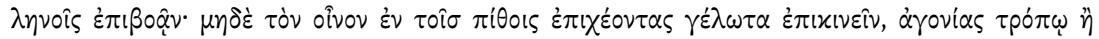

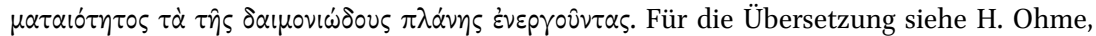
Concilium Quinisextum-Das Konzil Quinisextum (FC 82; Turnhout: Brepols, 2006) 255: „Ebenso bestimmen wir, keine komischen oder satyrischen oder tragischen Masken zu tragen und auch nicht den Namen des abscheulichen Dionysus anzurufen, während man die Trauben in den Keltern preßt; ebenso nicht beim Eingießen des Weines in Krüge in Gelächter auszubrechen und so aus Unkenntnis oder Leichtfertigkeit dämonischen Wahn hervorzurufen."

27) Vgl. hierzu grundsätzlich H. Noetzel, Christus und Dionysos: Bemerkungen zum religionsgeschichtlichen Hintergrund von Johannes 2,1-11 (Stuttgart: Calwer Verlag, 1960). 
Dionysos und Christus liefert uns ein Lehrstück in Sachen ,Inkulturation des Glaubens. “ "28

Auffällig ist, dass der Verfasser des Johannesevangeliums eine konkrete Auseinandersetzung mit Dionysos vermeidet, ${ }^{29}$ während beispielsweise die Konkurrenz durch eine direkte Überbietung bei Elia und den BaalsPriestern ${ }^{30}$ konkret fassbar ist. Im Vergleich dazu wird auch die Konkurrenz zwischen den Anhängern und den Gegnern Jesu klar und eindeutig im Evangelium thematisiert. In der Kana-Perikope soll „sich also Abwehr mit missionarischem Angriff ${ }^{\text {“31 }}$ verbinden. Die „scharfe Auseinandersetzung ${ }^{\text {“32 }}$ mit Dionysos wird mit stumpfer Klinge geführt, dionysischem Rausch steht „der Weinspender Jesus“ mit einem „überschäumenden Becher“33 gegenüber. Die konkurrierende Gottheit wird nicht erwähnt, sondern muss aus dem Text erschlossen werden.

\section{Die Krüge, der Wein und die Hochzeit}

Im Folgenden sind nun kurz die im Rahmen der Wundererzählung erwähnten Krüge, der Wein und die Hochzeit selbst zu diskutieren.

\subsection{Die Steinkrüge, ihre Funktion und ihr ursprünglicher Inhalt}

Die Geschichte vom Wunder bei der Hochzeit zu Kana erwähnt Wasserkrüge, die mit jüdischen Reinigungsriten in Verbindung stehen. ${ }^{34}$ Die 6

28) M. Theobald, Das Evangelium nach Johannes: Kapitel 1-12 (RNT; Regensburg: Friedrich Pustet, 2009) 218.

29) Vgl. E. Little, Echoes of the Old Testament in the Wine of Cana in Galilee (John 2:1-11) and the Multiplication of the Loaves and Fish (John 6:1-15): Towards an Appreciation (CRB 41; Paris: Gabalda, 1998) 50: „That there is no mention of Dionysos in the gospel, and no wedding in the Dionysos legend itself, causes Bultmann no second thoughts."

30) Vgl. 1 Kön 18.

31) C. Dietzfelbinger, Das Evangelium nach Johannes. Bd. 1:Johannes 1-12 (ZBK.NT 4/1; Zürich: Theologischer Verlag Zürich, 2001) 70.

32) Wengst, Johannesevangelium, 25 .

33) Vgl. jedoch E. Linnemann, „Die Hochzeit zu Kana und Dionysos: Oder das Unzureichende der Kategorien Übertragung und Identifikation zur Erfassung der religionsgeschichtlichen Beziehungen," NTS 20 (1974) 408-418, hier 416-417.

34) Vgl. R. Deines, Jüdische Steingefäße und pharisäische Frömmigkeit (WUNT 2/52; Tübingen: Mohr Siebeck, 1993). Siehe auch T. Nicklas, „Biblische Texte als Texte der Bibel interpretiert: Die Hochzeit zu Kana (Joh 2,1-11) in ,biblischer Auslegung," ZKTh 126 (2004) 241-256, hier 249-250. 
Krüge zu je 2 bis 3 Metreten (ca. 39 Liter ${ }^{35}$ scheint sich der Berichterstatter als Realien vorzustellen. ${ }^{36}$ Diese hatten angeblich „ein ungewöhnlich großes Fassungsvermögen." ${ }^{37}$

Die Hypothese, dass die Steinkrüge in ihrer Funktion erklärt werden müssten, ${ }^{38}$ weil die Adressaten des Johannesevangeliums nicht mit den jüdischen Sitten vertraut gewesen seien, ${ }^{39}$ wird durch die Erwähnung des Tafelmeisters, der in der rabbinischen Literatur unbekannt gewesen sei und das hellenistisch-christliche Milieu andeute, ${ }^{40}$ bestärkt.

Eine symbolische Deutung der religiösen Funktion der Reinigung ${ }^{41}$ ist meist von antijüdischen Topoi geprägt. ${ }^{42}$ Die Anzahl wird als Zeichen der Unvollkommenheit ${ }^{43}$ oder aber auch der Vollkommenheit (!) ${ }^{44}$ und das Ausgehen des Weines als fehlende Kraft des Judentums gedeutet. ${ }^{45} \mathrm{Zu}$ diesen Stereotypen gehört auch der Hinweis, dass nur die steinernen Krüge übrig sind, während „der Wein, der Geist der Freude, der von innen her belebt und erquickt, [...] ausgegangen" ist. ${ }^{46}$ Carson bemerkt in diesem Zusammenhang zu Recht, dass die Unvollkommenheit der sechs Krüge eigentlich durch einen siebten ausgeglichen werden müsste, um diese

35) Vgl. z.B. Thyen, Johannesevangelium, 157.

36) Vgl. auch E. Stauffer, „Historische Elemente im vierten Evangelium,“ in Bekenntnis zur Kirche: Festgabe für Ernst Sommerlath zum 7o. Geburtstag (Berlin: Evangelische Verlagsanstalt, 1960) 33-51, hier 34 .

37) U. Wilckens, Das Evangelium nach Johannes (18. Aufl./2. durchgesehene Aufl.; NTD 4; Göttingen: Vandenhoeck \& Ruprecht, 2000) 57.

38) So Nicklas, „Biblische Texte,“ 250; vgl. auch R.A. Culpepper, Anatomy of the Fourth Gospel: A Study in Literary Design (Philadelphia: Fortress Press, 1983) 221.

39) Vgl. z.B. L. Kierspel, The Jews and the World in the Fourth Gospel: Parallelism, Function and Context (WUNT 2/220; Tübingen: Mohr Siebeck, 2006) 26-27.

40) J. Gnilka, Johannesevangelium (NEB 4; Würzburg: Echter, 1983) 22.

41) Zumstein, „Bibel als literarisches Kunstwerk,“ $75^{-76}$.

42) Vgl. auch z.B. Collins, „Cana,“ 89 oder L. Morris, The Gospel According to John (2. Aufl.; Grand Rapids: Eerdmans, 1995) 155 .

43) Vgl. z.B. A.J. Köstenberger, John (Baker Exegetical Commentary on the New Testament; Grand Rapids: Baker Academic, 2004) 96; Moloney, Belief in the Word, 85 oder C.K. Barrett, Das Evangelium nach Johannes (KEK.S; Göttingen: Vandenhoeck \& Ruprecht, 1990) 215.

44) So Theobald, Evangelium nach Johannes, 214.

45) Vgl. Köstenberger, John, 93: „The running out of wine at the Cana wedding may be symbolic of the barrenness of Judaism.“

46) G. Kittel, Der Name über alle Namen II. Biblische Theologie NT (2. Aufl.; BTSP 3; Göttingen: Vandenhoeck \& Ruprecht, 1996) 153. 
Deutung als korrekt erscheinen zu lassen. ${ }^{47}$ Fraglich ist, ob die Zahlenangabe überhaupt symbolisch gedeutet werden muss. ${ }^{48}$ Es gibt auch Versuche, die Typologie "Gesetz“ versus "Gnade “49 in „vorbereitende Größe“ und „endzeitliche Vollendung “ umzudeuten und dabei die implizite Abwertung zu verneinen. ${ }^{50}$

Es scheint, hierfür spricht der archäologische Befund, ${ }^{51}$ dass diese Steingefäße zwar für die Zeit des Zweiten Tempels im Heiligen Land typisch waren, in anderen Gebieten des Römischen Reichs jedoch nur selten vorkamen. Damit würde die Hochzeitsgesellschaft den strengen jüdischen Reinheitsgesetzen folgen. ${ }^{52} \mathrm{Ob}$ deswegen auf eine Ablöse dieser Riten angespielt wird, darf bezweifelt werden. ${ }^{53}$ Das erwähnte Auffüllen der Krüge bis zum Rand macht einen Betrug unmöglich, ${ }^{54}$ somit beweisen die leeren Wassergefäße ein tatsächliches Wunder, da eine Vermischung des Wassers mit Wein ausgeschlossen ist.

\subsection{Der Wein}

Beim Wein geht es einmal um die Menge, dann um die Tatsache, dass Wasser zu Wein gewandelt wird sowie auch um die Qualität.

\subsubsection{Die Menge}

Sehr häufig wird die „Fülle“55 des Weins hervorgehoben. Die 6 Steingefäße fassten jeweils 2 bis 3 Metreten (dieses Hohlmaß entspricht etwa 40 Liter)

47) So D.A. Carson, The Gospel According to John (Leicester: Inter-Varsity, 1991) 174 und Morris, John, 161.

48) L.P. Jones, The Symbol of Water in the Gospel ofJohn (JSNT.S 145; Sheffield: Sheffield Academic Press 1997) 59: „[...] numbers seldom have symbolic meaning in the Fourth Gospel.“ 49) Siehe auch Hengel, „Der ,dionysische“ Messias, “ 588: „Daß die Antithese zwischen dem Wasser in den großen steinernen Gefäßen, die der rituellen Reinigung dienen, und dem guten Wein die Antithese im Prolog 1,17 weiterführt, ist seit der Alten Kirche immer wieder hervorgehoben worden."

50) Vgl. z.B. F. Zeilinger, Die sieben Zeichenhandlungen Jesu im Johannesevangelium (Stuttgart: Kohlhammer, 2011) 3 .

51) Vgl. Y. Magen, The Stone Vessel Industry in the Second Temple Period: Excavations at Hizma and the Jerusalem Temple Mount (Jerusalem: Israel Exploration Society, 2002) 162.

52) Magen, Stone Vessel, 141.

53) So z.B. Deines, Jüdische Steingefäße, 274.

54) Köstenberger, John, 97: „Filling the jars with water (to the top) proves the impossibility of deception or fraud."

55) So U. Busse, Das Johannesevangelium: Bildlichkeit, Diskurs und Ritual (BEThL 162; Leuven: University Press, 2002) 319; vgl. W.J. Bittner, Jesu Zeichen im Johannesevangelium: Die 
und enthielten somit zwischen rund 480 und 720 Liter. ${ }^{56}$ Wein, so scheint es, wird im Überfluss, ${ }^{57}$ in unvorstellbarer Menge ${ }^{58}$ geschenkt. Von einem „Luxuswunder"59 ist die Rede, die „ungeheuren Mengen Wein“60 seien "groß genug, um ein ganzes Bataillon Soldaten mehr als festlich zu bewirten,“61 ja von „nahezu unerschöpflichen Nahrungsmengen"62 wird geschrieben. Die „Riesenmenge von Wein veranschaulicht schon die Größe des zu erwartenden Wunders.“63 Dies wird als Überbietung anderer Weinwunder64 und „,bewußter Gegenentwurf“ zum Dionysos-Mythos“65 gedeutet. Auch die Erwähnung der jüdischen Reinigungsvorschriften wird als Teil einer „Überbietungstendenz" 66 gesehen. Ferner wird angemerkt,

Messias-Erkenntnis im Johannesevangelium vor ihrem jüdischen Hintergrund (WUNT 2/26; Tübingen: Mohr Siebeck, 1987) 115 Anm. 25; Collins, „Cana,“ 80. Siehe auch J. Becker, Das Evangelium nach Johannes: Kapitel 1-1o (3. Aufl.; ÖTBK 4/1; Gütersloh: Gütersloher, 1991) 131; E. Haenchen, Das Johannesevangelium: Ein Kommentar aus den nachgelassenen Manuskripten (hrsg. U. Busse; Tübingen: Mohr, 1980) 189; R. Bultmann, Evangelium des Johannes (20. Aufl.; KEK 2; Göttingen: Vandenhoeck \& Ruprecht, 1985) 82.

56) Vgl. Wengst, Johannesevangelium, 110-111.

57) Vgl. J.T. Nielsen, Die kognitive Dimension des Kreuzes: Zur Deutung des Todes Jesu im Johannesevangelium (WUNT 2/263; Tübingen: Mohr Siebeck, 2009) 258; Zumstein, „Bibel als literarisches Kunstwerk,“ 78; Thyen, Johannesevangelium, 157; Nicklas, „Biblische Texte,“ 249; vgl. auch F.J. Moloney, The Gospel of John (Sacra Pagina 4; Collegeville: The Liturgical Press, 1998) 68.

58) H. Thyen, „Das Johannesevangelium als literarisches Werk,“ in Studien zum Corpus Iohanneum (hrsg. H. Thyen; WUNT 214; Tübingen: Mohr Siebeck 2007 = zuerst abgedruckt in Teufelskinder oder Heilsbringer—die Juden im Johannes-Evangelium [hrsg. D. Neuhaus; ArTe 64; Frankfurt: Haag und Herchen, 1990] 112-132) 351-369, hier 367.

59) Vgl. Thyen, Johannesevangelium, 151; so auch R. Nordsieck, Das Geheimnis des Lazarus: Zur Frage nach Verfasser und Entstehung des Johannes-Evangeliums (Theologie 98; Münster/ Berlin: Lit-Verlag, 2010) 31 unter Verweis auf D.F. Strauß.

60) Siehe G.L. Borchert, John 1-11 (The New American Commentary 25A; Nashville: Broadman \& Holman, 1996) 156 oder Kittel, Name über alle Namen, 154.

61) Hirsch, Das vierte Evangelium, 123.

62) B. Kollmann, Ursprung und Gestalten der frühchristlichen Mahlfeier (GTA 43; Göttingen: Vandenhoeck \& Ruprecht, 1990) 207.

63) Zeilinger, Sieben Zeichenhandlungen, 27.

64) G. Theißen, Urchristliche Wundergeschichten: Ein Beitrag zur formgeschichtlichen Erforschung der synoptischen Evangelien (6. Aufl.; StNT 8; Gütersloh: Gütersloher Verlagshaus, 1990) 273 .

65) Riedl, Zeichen und Herrlichkeit, 280.

66) Vgl. Zeilinger, Sieben Zeichenhandlungen, 23. 
dass insgesamt ein mehrfaches Kontrastschema in dieser Erzählung zu erkennen sei. ${ }^{67}$

Ein weiteres mögliches Erklärungsmodell wäre der Wunsch der Adressaten, einmal auch „Wein in Fülle“ zu haben. ${ }^{68}$ Wegen dieser Fülle eines berauschenden Getränks ruft dieser Text "Verlegenheit" 69 hervor und wird sogar als anstößig ${ }^{70}$ empfunden; dies wird jedoch in Folge auch als verfehlte Deutung zurückgewiesen. ${ }^{71}$ Eine direkte Verbindung zwischen der Menge und einer möglichen Konkurrenz wird konstatiert, wenn von „wahrhaft dionysischen Weinmengen ${ }^{472}$ geschrieben wird. Ganz vereinzelt finden sich Stimmen, welche die Menge des Weins nicht als völlig übermäßig charakterisieren. ${ }^{73}$

\subsubsection{Die Wandlung}

Im Rahmen einer typologischen Auslegung kann die Aufhebung des Jüdischen durch das Christliche (teilweise auch unter Verwendung textkritischer Argumente) ${ }^{74}$ betont werden.$^{75}$ Man versucht, dieses antijüdische Stereotyp abzuschwächen, indem von Kontinuität ${ }^{76}$ oder von „Transformation jüdischer Riten "77 gesprochen wird, auch wenn dies in keiner Weise durch den Text selbst gerechtfertigt ist. Die Steingefäße sind Behältnisse für eine Flüssigkeit, deren Inhalt sich ändert. Es findet sich kein Hinweis

67) R. Zimmermann, Christologie der Bilder im Johannesevangelium: Die Christopoetik des vierten Evangeliums unter besonderer Berücksichtigung von Joh 10 (WUNT 171; Tübingen: Mohr Siebeck, 2004) 205: „Das Kontrastschema (erst—dann; alt—neu), [...] kann m.E. als grundlegendes Strukturmuster der gesamten Erzählung betrachtet werden: Dem Weinmangel wird die Weinfülle entgegengesetzt, weniger guter Wein wird durch guten Wein kontrastiert, was der ,schlechte‘ Bräutigam versäumt, wird durch Jesu Handeln überboten, dem Unwissen des Weinschenks steht das Wissen der Diener gegenüber usw.“

68) Wengst, Johannesevangelium, 112.

69) Wengst, Johannesevangelium, 105.

70) So Little, Echoes, 10.

71) Nicklas, „Biblische Texte,“ 254.

72) Vgl. P. Pilhofer, „Dionysos und Christus. Zwei Erlöser im Vergleich,“ in Die frühen Christen und ihre Welt. Greifswalder Aufsätze 1996-2001. Mit Beiträgen von Jens Börstinghaus und Eva Ebel (hrsg. P. Pilhofer; WUNT 145; Tübingen: Mohr Siebeck, 2002) 73-91, hier 83.

73) Little, Echoes, 51: „It is a generous quantity for a country wedding but pitiful in terms of an eschatological vision."

74) Vgl. J. Ashton, Understanding the Fourth Gospel (2. Aufl.; Oxford: Oxford University Press, 2008) 175 Anm. 70.

75) Wilckens, Evangelium nach Johannes, 58.

76) Nicklas, „Biblische Texte,“ 253.

77) Moloney, Belief in the Word, 8 o. 
darauf, dass diese Gefäße nun nicht mehr verwendet werden. Gerade weil sich auch antijüdische Aussagen im Johannesevangelium belegen lassen, wäre es für eine solche Interpretation unabdingbar, sie auch im konkreten Text nachzuweisen. Aus der Wandlung des Wassers in Wein wird auch ein HinweisaufdieGöttlichkeitJesuund "seineVollmachtüberdieSchöpfung“78 abgeleitet.

\subsubsection{Die Qualität}

Der Architriklinos hebt gegenüber dem Bräutigam die Qualität hervor. Dabei könnte es sich um einen Beleg dafür handeln, dass Jesu Werke „außerordentlich" 79 sind. Man hat in diesem Zusammenhang auch darauf hingewiesen, dass der Wein, der als Gabe des messianischen Äons gesehen werden kann, ${ }^{80}$ auch eine entsprechende Qualität haben muss. ${ }^{81}$ Eine Verbindung zum Anbruch des messianischen Zeitalters wird auch mit Hilfe der Fülle des Weins hergestellt. ${ }^{82}$ Im Zusammenhang dieses Verses wird ferner auf die - sonst in der Antike nicht begegnende ${ }^{83}$ - so genannte „Weinregel“ verwiesen, die vom Architriklinos zitiert wird. ${ }^{84}$ Wein wurde in der Antike nicht ungemischt getrunken. ${ }^{85}$ Deswegen scheint der eigentliche Sinn der Aussage des Architriklinos darin zu bestehen, als „unverdächtiger Zeuge ${ }^{486}$ zu bestätigen, dass es sich um guten Wein ${ }^{87}$ und nicht um eine manipulierte Flüssigkeit wie z.B. gefärbtes Wasser handelt. ${ }^{88}$

Wenn man einmal von der Frage, ob es die so genannte Weinregel überhaupt gab, absieht, scheint man annehmen zu dürfen, dass mit dieser

78) Zumstein, „Bibel als literarisches Kunstwerk,“ 72.

79) Köstenberger, John, 98.

80) W. Grundmann, „Verkündigung und Geschichte in dem Bericht vom Eingang der Geschichte Jesu im Johannes-Evangelium," in Der historische Jesus und der kerygmatische Christus: Beiträge zum Christusverständnis in Forschung und Verkündigung (hrsg. H. Ristow und K. Matthiae, Berlin: Evangelische Verlagsanstalt, 1960) 289-309, hier 296-297.

81) Collins, „Cana,“ 89.

82) Wengst, Johannesevangelium, 112; Nicklas, „Biblische Texte,“ 251.

83) Siehe hierzu auch Zeilinger, Sieben Zeichenhandlungen, 28.

84) Vgl. z.B. Wengst, Johannesevangelium, 112. Dagegen jedoch Wilckens, Evangelium nach Johannes, $57:$ „[...] das ist natürlich keine,Weinregel', weder damals noch heute!“

85) R.J. Forbes, Studies in Ancient Technology. Bd. 3 (2. Aufl.; Leiden: Brill, 1965) 80.

86) Thyen, Johannesevangelium, 159.

87) Wilckens, Evangelium nach Johannes, 57-58; Zumstein, „Bibel als literarisches Kunstwerk, “73-74; Zeilinger, Sieben Zeichenhandlungen, 27, und U. Schnelle, Das Evangelium nach Johannes (3. Aufl.; ThHK 4; Leipzig: Evangelische Verlagsanstalt, 2004) 71.

88) Vgl. Theißen, Urchristliche Wundergeschichten, 112-113. 
Formulierung grundsätzlich die in der Antike bekannte Tatsache kommentiert wird, dass es verschiedene Qualitätsstufen von Weinen in der Antike gab. Eine grundsätzliche Einteilung unterscheidet zwischen einem Wein, der relativ rasch verbraucht werden musste, und einem, der sich für eine längere Lagerung eignete. ${ }^{89}$ Ferner ist bekannt, dass Wein ein Lebensmittel darstellte, das oftmals wegen Mängeln beanstandet wurde, ${ }^{90}$ und dass über die Qualität höchst unterschiedliche Urteile existierten. ${ }^{91}$ Wein unterlag deswegen auch mannigfachen Manipulationen in der Antike. So konnte er mit diversen Gewürzen und Geschmacksstoffen versetzt werden, ${ }^{92}$ wobei hierzu auch aus heutiger Sicht eher merkwürdige Substanzen wie Meerwasser, Aloe oder Pfeffer gehörten. ${ }^{93}$

„Wein gehört—-neben Getreide, Öl und Hülsenfrüchten—zu den Grundnahrungsmitteln [...] der antiken Bevölkerung ${ }^{494}$ und wurde auch in minderer Qualität getrunken. Dass Wein gemischt und nicht rein getrunken wurde, liegt wohl daran, dass "der Wein in der Antike anscheinend einen relativ hohen Gehalt an Alkohol hatte (15-16 Vol.\%). “95 Durch die Aussage des Architriklinos wird also bestätigt, dass nicht Wein in den Wasserkrügen gemischt, sondern vielmehr Wasser in Wein verwandelt und reiner Wein zur Verkostung gereicht wurde. Das Wunder wird durch die Aussage des Architriklinos bestätigt. ${ }^{96}$ Man sollte die Funktion des Architriklinos

89) Vgl. É. Jakab, Risikomanagement beim Weinkauf: Periculum und Praxis im Imperium Romanum (MBPF 99; München: C.H. Beck, 2009) 23.

90) Jakab, Risikomanagement, 146: „Bis zum Ablauf der Garantiefrist konnte der Käufer den Wein kosten und beim Feststellen der relevanten Fehler (oxos, ozomenos, apoietos) zurückweisen." Siehe auch S. Hodeček, „,Vinum laetificat cor hominis‘-,Wein erfreut des Menschen Herz؛ Wein, Weinanbau und Weinkultur im antiken Ägypten, "in Mit den Griechen zu Tisch in Ägypten (2. Aufl.; hrsg. H. Froschauer und C. Römer; Nilus 12; Wien: Phoibos, 2009) 53-6o, hier $55^{-5} 6$.

91) Forbes, Studies, 78: „The classical authors did not consider the Egyptian wines worth mentioning. MARTIAL declares that vinegar tasted better. STRABO says that Libyan wine tastes well when mixed with sea-water, it will not cause headache but helps digestion."

92) Forbes, Studies, 73.

93) R. Frankel, Wine and Oil Production in Antiquity in Israel and Other Mediterranean Countries (American Schools of Oriental Research 10; Sheffield: Sheffield Academic Press, 1999) 43 und Appendix 2. Siehe auch Hodeček, „Vinum laetificat,“ 55 .

94) Hodeček, „Vinum laetificat,“ 53; siehe auch C.E. Walsh, The Fruit of the Vine: Viticulture in Ancient Israel (HSM 6o; Winona Lake: Eisenbrauns, 2000) 210; Frankel, Wine and Oil Production, 38 .

95) Hodeček, „Vinum laetificat,“ 57.

96) Fortna, Fourth Gospel, 50. 
allerdings auch noch im Kontext des vorliegenden Wunders stärker verankern. Die Hochzeit war bereits im Gange, die Gäste sind, dies wird auch aus der Antwort des Architriklinos deutlich, nicht mehr nüchtern. Und gerade in einem derartigen Fall ist ein nüchterner Zeuge-in diesem Fall eben gerade der Architriklinos-notwendig, damit nicht nachträglich der Einwand erhoben werden kann, dass Betrunkene getäuscht worden seien. ${ }^{97}$ Schließlich sind ja gerade Betrunkene oftmals Opfer betrügerischer Handlungen. ${ }^{98}$

\subsection{Die Hochzeit}

Dass bei der Hochzeit der Wein ausgeht, wird teilweise als Zeichen dafür gesehen, dass es sich um eine Hochzeit in einer sozial eher benachteiligten Schicht der Bevölkerung gehandelt haben dürfte. ${ }^{99}$ Dagegen spricht jedoch der Umstand, dass Speisemeister und Diener erwähnt werden. ${ }^{100}$ Die Tatsache, dass das Wunder bei einer Hochzeitsfeier geschieht, macht es möglich, Beziehung zur symbolischen Verwendung der Hochzeit als Bild für die Beziehung zwischen Gott und seinem Volk im Sinne eines Bundes herzustellen, ${ }^{101}$ wobei bei der Hochzeit auch intertextuelle Bezüge ${ }^{102} \mathrm{zu}$

\footnotetext{
97) Siehe hierzu jedoch Barrett, Evangelium nach Johannes, 216: „Für Schlußfolgerungen im Hinblick auf den Grad der Trunkenheit der Gäste bei dieser Hochzeit gibt es hier selbstverständlich keinen Grund.“

98) Vgl. hierzu H. Förster, ,, Streck dich nicht mit einer Verheirateten zum Weingelage hin (Sir 9,9a): Edition von P. Vindob. K. 9670," ZAC 14 (2010) 273-305, hier 282-283.

99) So Gnilka, Johannesevangelium, 22-23 und Morris, John, 162.

100) Bergler, Kana in Galiläa, 4.

101) Vgl. z.B. Nicklas, „Biblische Texte,“ 246: „An verschiedenen Stellen des Alten Testaments wird der Bund Gottes mit seinem Volk Israel mit dem Bild der ,Hochzeit' umschrieben (z.B. Hos 2,18-22 LXX; Jes 54,4-8; 62,4-5) —eine Symbolik, mit der zusätzlich der Gedanke verbunden ist, dass Gott in der Endzeit den Bund mit seinem Volk erneuern wird." Vgl. auch Kittel, Name über alle Namen, 153 .

102) Intertextualität wird oftmals erst vom Leser konstruiert, selbst wenn diese nicht vom Autor intendiert war; vgl. S. Gillmayr-Bucher, „Intertextuality: Between Literary Theory and Text Analysis," in The Intertextuality of the Epistles: Explorations of Theory and Practice (hrsg. T.L. Brodie, D.R. MacDonald und S.E. Porter; New Testament Monographs 16; Sheffield: Sheffield Phoenix Press, 2006) 13-23, hier 16-17: „Thus, a relation might either not be recognized by the reader or be recognized but not intended by the author. With the focus on the interrelations of texts, it is obvious that even if a relation comes into existence by chance, this relation starts to exist as soon as a reader recognizes it. Moreover, the general intertext of author and reader can be different, offering different choices of texts [...].“
} 
anderen Evangelien hergestellt werden. ${ }^{103}$ Die eschatologische Bedeutung des Wunders kann dabei in den Vordergrund rücken. ${ }^{104}$ „Das Signal, das so in der Erzählung vom Weinwunder in Kana gegeben wird, lautet daher, dass die messianische Zeit angebrochen ist, dass ,Jesus durch dieses Wunder sich als den zu erkennen gibt, der die Heilszeit bringt.' "105 Dies wird, so scheint es, gerade auch durch den Anlass des Wunders bestätigt: Eine Hochzeit ist einerseits ein freudiges Ereignis, andererseits hat die Hochzeit eine tiefe symbolische Bedeutung. ${ }^{106}$ Auch Anspielungen auf die eucharistische Mahlfeier werden gesehen. ${ }^{107}$ Umgekehrt wird festgehalten, dass es erstaunlich sei, dass die Reaktion der Gäste auf dieses Wunder nicht thematisiert wird. ${ }^{108}$ „Das heißt, es ist auch die ,novellistische, 'Spannung erregende Ausgestaltung der Erzählung in sich ernst zu nehmen, etwa der so frappierende, zum Teil Kopfschütteln erregende Schluß V. 1ob, mit dem die Hochzeitserzählung abrupt abbricht, um die kühne Metabasis zum Glauben der Jünger zu vollziehen. “109 Dass „die Hauptpersonen einer Hochzeit, Braut und Bräutigam, eher Statistenrollen einnehmen, die Braut wird nicht einmal erwähnt, "${ }^{110}$ ist höchst auffällig.

\section{Zusammenfassung der Diskussion}

Um den Stand der Überlegungen kurz zusammenzufassen: Die bisherige Auslegung fokussiert auf zwei mögliche Deutungen des Weinwunders bei

103) Nicklas, „Biblische Texte,“ 246 : „Zu erinnern wäre an die Parabel vom königlichen Hochzeitsmahl Mt 22,1-4, in der der Anbruch der Gottesherrschaft mit einem endzeitlichen Gerichtshandeln Gottes, aber auch dem Bild der Hochzeit verbunden wird."

104) Vgl. Zimmermann, Christologie der Bilder, 206-207: „Noch deutlicher dürfte die Weinfülle innerhalb der Symboltradition Israels als Hinweis auf die kommende Heilszeit betrachtet worden sein. [...] Wie der Wein, so ist auch die Hochzeit ein zentrales Symbol jüdischer Heilserwartung [...]." Siehe auch U. Wilckens, „Maria, Mutter der Kirche-Joh 19,26f.," in Der Sohn Gottes und seine Gemeinde: Studien zur Theologie der Johanneischen Schriften (hrsg. U. Wilckens; FRLANT 200; Göttingen: Vandenhoeck \& Ruprecht, 2003) 147-166, hier 151 (= Ekklesiologie des Neuen Testaments. FS K. Kertelge [Freiburg: Herder, 1996] 247-266).

105) Wengst, Johannesevangelium, 112.

106) Zumstein, „Bibel als literarisches Kunstwerk,“ 78 : „Im unmittelbaren Sinn ist die Hochzeit zu Kana Ort der Lebendigkeit, der Freude und der Liebe, aber gleichzeitig wird dadurch der Anbruch der Endzeit als Heilszeit angedeutet."

107) Wilckens, Evangelium nach Johannes, 59.

108) Vgl. Moloney, Belief in the Word, 91.

109) Hengel, „Der ,dionysische‘ Messias, “ 585 .

110) Zimmermann, Christologie der Bilder, 205-206. 
der Hochzeit zu Kana. Einerseits scheint es, dass durch die „Menge des guten Weines" ein auch von Dionysos bekanntes Wunder überboten werden kann. Andererseits ist es durch dieses Wunder auf einer Hochzeit, so scheint es, möglich, Bezüge zur Hochzeit als Symbol des himmlischen Reiches herzustellen. Problematisch an beiden Deutungen ist, dass in beiden Fällen im Rahmen der Exegese Implizites explizit gemacht werden muss: Im Fall der Deutung der Hochzeit als Anspielung auf die eschatologische Existenz muss vorausgesetzt werden, dass der vom Verfasser implizierte Leser diesen Bezug tatsächlich herstellen konnte, obwohl gerade nicht auf diese symbolische Deutung explizit hingewiesen wurde. Bereits die Erwähnung einer Hochzeit würde also als Symbol auf die eschatologische Bedeutung hinweisen und in diesem Fall ein "allseits bekanntes“ Symbol darstellen, das nicht weiter erklärt werden müsste. ${ }^{111}$ Intratextuell ist es jedenfalls die einzige Erwähnung einer Hochzeit im Johannesevangelium. Das wirft die Frage auf, ob die intertextuellen Bezüge tatsächlich intendiert waren. Die Angabe eines konkreten Ortes mahnt zur Vorsicht bei einer symbolischen Interpretation des Geschehens. Der Bezug zum Dionysoskult ist im Vergleich zu der im ersten Buch der Könige berichteten Auseinandersetzung um den Baalskult auf der einen und zur Behandlung der Juden durch den Verfasser des Johannesevangeliums auf der anderen Seite als „höchst diplomatisch“ zu bezeichnen. Dies wird bei einer Betrachtung der papyrologischen Zeugnisse noch deutlicher.

\section{Archäologische und papyrologische Evidenz für Wein, Weinlieferungen und Weinqualität}

Nach herrschender Meinung der Exegese, so darf festgehalten werden, handelt es sich bei der Weinmenge von ca. 500 bis 700 Liter um eine unglaublich große Menge an Wein. Um dies in den Kontext der archäologischen Situation zu stellen, sei einleitend Éva Jakab etwas ausführlicher zitiert, welche die Situation des antiken Weinguts knapp und konzise beschreibt: „Cato empfiehlt, ein Weingut auf etwa 100 iugera anzulegen; der durchschnittliche landwirtschaftliche Betrieb in der ausgehenden Republik und des Prinzipats war jedoch der fundus, dessen Größe zwischen

111) Gillmayr-Bucher, Intertextuality, 19: „But especially references to supposedly,well known literature often lack such markers. Consequently, the recognition depends on the textual knowledge of the reader, and it is easily imaginable that references may pass unnoticed." 
25 und 50 iugera lag. Ein Weingut dieser Größe dürfte auch zu der Villa della Pisanella in Boscoreale, in der Nähe von Pompeji, gehört haben, deren Keller mit 84 dolia, mit einem Fassungsvermögen von insgesamt 84.000 bis 110.000 Liter, ausgestattet war. ${ }^{112}$ Aus dem Gebiet des heutigen Armenien sind sogar noch größere Weinlager aus vorchristlicher Zeit bekannt. ${ }^{113}$ Dolia dienten zur Aufbewahrung von Vorräten. Dazu zählen Wein, Öl und Getreide, wobei die Dolia oftmals im Boden versenkt waren. Teilweise wurden sie auch als Transportgefäße verwendet. „Bei der Weinproduktion macht der Wein in der cella vinaria im d(olium) die Gärung durch. ${ }^{114}$ Auch müsste man dafür, dass die gewandelte Weinmenge vom antiken Leser als „übermäßig“ angesehen wurde, annehmen, dass keiner der Leser der Perikope von der Hochzeit zu Kana jemals das Auffangbecken einer durchschnittlichen Weinpresse in Palästina gesehen hatte, dessen kleinere Ausführungen durchschnittlich rund 24 Hektoliter fassten-wobei Auffangbecken mit bis zu 590 Hektolitern bezeugt sind. ${ }^{115}$ Dies ist jedoch unwahrscheinlich, hatte doch beispielsweise das kleine Städtchen Dor, dessen Einwohnerzahl in der Spätantike auf nicht mehr als 2500 Einwohner geschätzt wird, 30 Weinpressen, deren Auffangbehälter ein kumuliertes Volumen von rund 230.000 Litern besaßen. ${ }^{116}$

Der auf einem normalen Weingut des Römischen Reiches gekelterte Wein konnte ohne Probleme die Größenordnung von bis zu 1000 Hektolitern erreichen. So könnte man auf SB XVI 12486 (aus dem ägyptischen Verwaltungsbezirk Hermopolites; datiert auf 3. Phaophi [d.i. 30. September] 470 ) verweisen. Nach diesem Weinlieferungskauf wurden 250 knidia, also zwischen 550 und 1100 Litern, Wein gekauft. Der Vertrag wurde am 30. September 470 mit Lieferungstermin des Weins für Mesore (Juli/August) 471

112) Vgl. Jakab, Risikomanagement, 5 .

113) Vgl. Forbes, Studies, 73: „Recent excavations in this region at Karmir-Blur (Teishebaini) have yielded remains of very large storage-rooms, where no less than 150,000 litres of wine was kept."

114) Vgl. R. Docter, „Dolium,“ Der Neue Pauly 3 (1997) 734.

115) Vgl. S.A. Kingsley, A Sixth-Century ad Shipwreck off the Carmel Coast, Israel: Dor D and Holy Land Wine Trade (British Archaeological Reports. International Series 1065; Oxford: Archaeopress, 2002) 71 (der Studie liegen 186 Weinpressen aus der späten römischen sowie der byzantinischen Zeit aus Palästina zugrunde): „If the vat of an average-sized installation was full, a single pressing could have produced just over 2400 litres of wine (105 amphorae). A relatively large number of the installations ( 76 out of $186: 42 \%$ of the sample) exceed the average size and may be defined as ,large scale' presses whose vats hold between 4000 and 59,000 litres (174 and 2565 amphorae [...]).“

116) Kingsley, Shipwreck, 73. 
geschlossen. ${ }^{117}$ Dieser Eindruck, dass die in der Perikope von der Hochzeit zu Kana erwähnte Weinmenge nicht übermäßig groß gewesen ist, wird auch durch andere Weinlieferungskäufe bestätigt. So erwähnt beispielsweise P.Eirene II 7 genau 250 kadoi (etwa 500 Liter) ${ }^{118}$ und P.Flor. I 65 etwa 1000 Liter. ${ }^{119}$ Größere Weinmengen-der größte papyrologisch belegte Weinlieferungskauf betrifft ein Volumen von ca. 8.19o Litern-wurden wohl deswegen gekauft, um sie (zumindestens teilweise) weiterzuverkaufen. ${ }^{120}$ Diese Mengen sollten auch auf dem Hintergrund des Weinverbrauchs in Palästina in der Spätantike gesehen werden: Nach modernen Schätzungen wurden jährlich 73 Millionen Liter unverdünnter Wein in diesem Gebiet verbraucht-dies entspricht dem 100.00o-fachen der in Kana gewandelten Menge. Auch die dafür benötigten rund 3,2 Millionen Amphoren mit einer Füllmenge von ca. 23 Litern zeigen, wie groß der Verbrauch damals war. ${ }^{121}$

Die im Bibeltext erwähnte Weinmenge als „haushaltsüblich“ zu bezeichnen, ${ }^{122}$ scheint auch vor dem Hintergrund des aus der Antike überlieferten Verbrauchs sinnvoll. Marcus Porcius Cato ${ }^{123}$ gibt nach der Berechnung von Dieter Flach im Rahmen seines Werkes De agri cultura die jährliche Weinmenge für einen erwachsenen Sklaven mit rund 1,84

\footnotetext{
117) Zu dem Papyrus vgl. Jakab, Risikomanagement, 123.

118) Siehe auch Jakab, Risikomanagement, 140.

119) Jakab, Risikomanagement, 142.
}

120) Vgl. S. Hodeček und F. Mitthof, „Ein Weinlieferungskauf aus dem Herakleopolites, “ $A P F$ 51 (2005) 76-86, hier 76-77: „Ein gewisser Flavius Pseei [...] kauft von einem Einwohner des Dorfes Papa Mikra namens Aurelius Apollos 2.500 Dipla Wein. Das Fassungsvermögen der Dipla betrug sechs Sextare; die Liefermenge entsprach somit, den Sextar zu o,546 l gerechnet, 8.19o l. Dieses Volumen ist für Weinlieferungskäufe ungewöhnlich groß [...] und dürfte den Eigenbedarf des Pseei [...] deutlich überstiegen haben. "Siehe zu diesem Text jetzt auch Jakab, Risikomanagement, 149.

121) Kingsley, Shipwreck, 61: „[...] it is conceivable that Palestine may have required somewhere in the range of at least 73 million litres of undiluted wine annually in Late Antiquity (about 3.2 million amphorae of 23 litre average capacity).“

122) Siehe hierzu auch S. Dar, Landscape and Pattern: An Archaeological Survey of Samaria 8 oo B.C.E. - 636 C.E. With a Historical Commentary by Shimon Applebaum. Part 1 (BAR International Series 308[i]; Oxford: B.A.R., 1986) 161: „According to the above rulings, the normal person was in the habit of obtaining and storing five oil jars and 15 wine jars during the year [...]. Calculations of the amounts of wine according to the capacity of the amphorae (22-25 litres approximately), indicate that a quantity of 330-375 litres of wine were (sic!) brought home."

123) M. Porcius Cato: 234-149 v.Chr.; Konsul im Jahre 195; Censor im Jahr 184; zu Leben und Werk vgl. A.E. Astin, Cato the Censor (Oxford: Clarendon Press, 1978). 
Hektolitern an ${ }^{124}$ - es sollte jedoch nicht verschwiegen werden, dass auf Cato aufbauend auch größere Mengen errechnet wurden. ${ }^{125}$ Fritz Mitthof veranschlagt den täglichen Verbrauch der unteren, grundbesitzlosen Schichten mit rund 0,2 Litern pro Tag, ${ }^{126}$ was einem Verbrauch von gut 0,7 Hektolitern pro Jahr entsprechen würde. Selbst bei o,7 Hektolitern pro Jahr würde die maximale Menge der in Kana gewandelten Flüssigkeit nur in etwa dem Jahresbedarf von 10 Erwachsenen entsprechen-und dass in Palästina in der Antike ein ähnlicher Weinverbrauch wie im gesamten Gebiet des Mittelmeers üblich war, ist bekannt. ${ }^{127}$ Und da Wein oftmals auch als Jahreslieferung gekauft bzw. geliefert wurde, wird man wohl die Menge bei der Hochzeit zu Kana tatsächlich als „haushaltsübliche“ Menge bezeichnen dürfen, so dass die Beschreibung der Menge als „Übermaß“ und „unvorstellbare Fülle“ als sachlich unzutreffend abzulehnen ist, auch wenn diese Behauptung häufig wiederholt worden ist. ${ }^{128}$ Wenn man die Berechnung von Carey Ellen Walsh zugrunde legt, dass aus den Angaben von Cato ein jährlicher Pro-Kopf-Verbrauch von 26o Litern abzuleiten wäre und dass der Weinverbrauch in Palästina tendenziell höher war als von Cato angegeben, ${ }^{129}$ würden die rund sieben Hektoliter, auf die man käme, wenn für alle sechs Gefäße drei Metreten angesetzt werden, nicht einmal den

124) Für die Berechnung auf Grundlage von Cato, De agri cultura 57 vgl. D. Flach, hrsg., Marcus Porcius Cato. Über den Ackerbau (Stuttgart: Franz Steiner Verlag, 2005) 141 Anm. 68.

125) Walsh, Fruit of the Vine, 210-211: „Cato advised that for the family farm, each field worker be given as much as ten amphorae of wine a year. This works out to be approximately 260 litres or 347 bottles of the .75 l size (today's standard bottle) a year, almost a bottle a day."

126) F. Mitthof, „Das Lebensnotwendige: Grundnahrungsmittel, Rationen, Preise,“ in Mit den Griechen zu Tisch in Ägypten (2. Aufl.; hrsg. H. Froschauer und C. Römer; Nilus 12; Wien: Phoibos, 2009) 21-28, hier 27.

127) Walsh, Fruit of the Vine, 210.

128) Siehe hierzu grundsätzlich auch H. Hauben, „Christ versus Apollo in Early Byzantine Kourion? With a Note on the So-called ,Panayia Aphroditissa in Paphos," in Philomathestatos: Studies in Greek and Byzantine Texts Presented to Jacques Noret for His Sixty-Fifth Birthday (hrsg. B. Janssens, B. Roosen und P. van Deun; OLA 137, Leuven/Paris/Dudley: Peeters, 2004) 269-284, hier 281-282: „The [...] study reminds us of the necessity to handle matters of religious history with extreme care, especially when being confronted with at first glance unexpected or strange developments. More than in other circumstances, fantasy should be firmly restrained to avoid that eagerly believed and increasingly repeated misunderstandings go their own way before ending up as quasi established fact.“

129) Walsh, Fruit of the Vine, 211: „Since the agricultural strategies for ancient Israel were similar, in its mixed farming of grains, grapes, and olives, we reasonably infer from analogy that the Israelite's wine consumption would approach, or even surpass, that of a Roman farmhand." 
jährlichen Verbrauch von drei (!) Erwachsenen decken. Mittelalterliche Vergleiche zeigen, dass die Schätzung von Cary Ellen Walsh wohl zu hoch angesetzt ist, während die von Dieter Flach ${ }^{130}$ oder auch die von Sean Kingsley vorgeschlagene Menge glaubhaft erscheint. ${ }^{131}$ Deswegen scheint die angeblich für das fröhliche Fest des Bataillons, das bereits erwähnt wurde, ausreichende Weinmenge wohl nicht einmal für die alltägliche Bedarfsdeckung der in Palästina stationierten Soldaten zu reichen: Der tägliche Bedarf des römischen Heeres in Palaestina Tertia wird auf bis zu knapp 1400 Liter geschätzt ${ }^{132}$ _ dies würde der durchaus realistischen Menge von knapp 0,4 Litern pro Person und Tag entsprechen. Deswegen sollte in diesem Zusammenhang erwähnt werden, dass Wein letztlich ein Grundnahrungsmittel war, das wenigstens ein Viertel der täglich verbrauchten Kalorien bereitstellte. ${ }^{133}$

\section{Wein als Zeichen des Festes}

Der bei der Hochzeit zu Kana gewandelte Wein wird als Zeichen des Festes gesehen. Hier scheint der moderne Weinverbrauch, der beispielsweise für Israel nicht einmal mehr ein Vierzigstel des pro Kopf in der Antike verbrauchten Weins ausmacht, die Wahrnehmung des Weins als eines Festgetränks beeinflusst zu haben. ${ }^{34}$ Schließlich setzt auch der Titus-Brief die Verfügbarkeit dieses Getränks in einer Menge voraus, dass der Gebzw. Missbrauch durch Greisinnen thematisiert wird. ${ }^{135}$ Allerdings muss

130) Flach, Cato, 141 Anm. 68.

131) Kingsley, Shipwreck, 61: „Tchernia's detailed study of historical texts and comparative sources from Italy and France, dating between the 14th and 19th centuries (1986: 26), concluded that men consumed between 146 and 182 litres of undiluted wine every year in the Roman West and that intake levels amongst women comprised half that amount."

132) Kingsley, Shipwreck, 61: „If Palestine was also responsible for meeting the demands of the annona militaris by supplying wine to the two legions stationed in Palaestina Tertia, each composed of about $175^{\circ}$ men [...], an additional 22,000 full amphorae would have been added to this burden."

133) Kingsley, Shipwreck, 6o: „Wine was a staple source of nutrition in antiquity that provided at least one-quarter of the daily caloric intake and about one-third of the body's iron requirements according to current estimates [...]."

134) Kingsley, Shipwreck, 61: „Unlike in recent times, when only an estimated 4.2 litres is drunk annually per capita in Israel for social reasons and personal pleasure [...], wine's basic nutritional value generated a ,massive' demand in the Roman and Byzantine Periods."

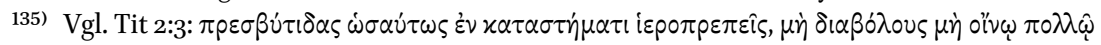

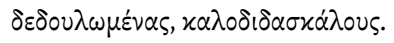


angemerkt werden, dass es ein eher neuer Trend ist, für die Antike tatsächlich den Nährwert von Wein und Öl für die Ernährung zu berücksichtigen, ${ }^{136}$ so dass dies wohl zu erklären vermag, dass die Menge und der Nährwert des in der Antike verbrauchten Weins bisher in der Diskussion zu wenig thematisiert wurden. ${ }^{137}$ Und so scheint es ein modernes Missverständnis zu sein, anzunehmen, dass das Weintrinken beim letzten Mahl Jesu (Mk 14,23.25 par) auf ein besonderes Mahl hinweise, „da man sonst nur Wasser trank. “138 Schließlich sei Wein nur ,an festlichen Gelegenheiten“139 getrunken worden. Dies lässt sich bis in liturgische Texte hinein verfolgen, ${ }^{140}$ darf jedoch als Teil der Wirkungsgeschichte gesehen werden, die in dieser Frage den historischen Gegebenheiten nicht gerecht wird.

\section{Ergebnis}

Auf dem Hintergrund dieser Überlegungen muss die Frage gestellt werden, was das Ziel dieser Wundererzählung ist. Selbstverständlich kann man argumentieren, dass es sich um ein "Geschenkwunder"141 handelt. Allerdings wäre die Frage zu stellen, warum in diesem Fall der Akt der

136) Vgl. P. Horden und N. Purcell, The Corrupting Sea: A Study of Mediterranean History (Oxford: Blackwell, 2002) 203: „Much more important, it is only relatively recently, and thanks in part to the ethnological approach once again, that the crucial nutritional role played in the Mediterranean diet by oil and wine themselves has begun to be recognized [...]."

137) Vgl. jedoch auch nur E. Haag, „Wein. I. Biblisch,“ LThK 10 (3. Aufl.; 2001) 1027-1028, hier 1027: „Zur vollen Mahlzeit gehörte der W. zus. mit dem Brot (Gen 14,18; 1 Sam 16,20; 25,18) als Grundnahrungsmittel (Gen 27,25; Ri 19,19; Sir 39,26). Als Genußmittel fehlte der W., der Götter u. Menschen erfreut (Ri 9,13; Ps 104,15; Koh 10,19; Sir 31,27), bei keinem festl. Anlaß (Ri 14,10; 1 Chr 12,40; 1 Makk 16,15); er galt als Heilmittel (Lk 10,34) z. Überwindung v. Traurigkeit (Koh 2,3) u. z. Vergessen v. Mühsal u. Sorgen (2. Sam 16,2; Spr 31,6f.).“

138) R. Feneberg, Christliche Passafeier und Abendmahl: Eine biblisch-hermeneutische Untersuchung der neutestamentlichen Einsetzungsberichte (StANT 27; München: Kösel-Verlag, 1971) 17 .

139) J. Jeremias, Die Abendmahlsworte Jesu (4. Aufl.; Göttingen: Vandenhoeck \& Ruprecht, 1967) 44-45.

140) Vgl. E.L. Fellechner und H. Miethe, Praxishilfe Gottesdienstliturgie: Advent bis Pfingsten. Bd. 1 (Nidderau: Verlag Neues Buch, 1997) 422; dort heißt es im Gebet bei der Vorbereitung der Gaben für den Pfingstsonntag: „Der Wein ist Zeichen der Freude, des Festes-Zeichen für das Reich Gottes." Vgl. auch R. Christiansen und P. Cornehl, hrsg., Alle an einen Tisch: Forum Abendmahl 2 (Gütersloh: Gütersloher Verlagshaus Gerd Mohn, 1981) 106: „Herr, unser Gott, wir loben dich. Du schenkst uns die Frucht des Weinstocks, das Zeichen des Festes.“ 141) Zimmermann, Christologie der Bilder, 204. 
Schenkung keine zentralere Stellung innerhalb der Wundergeschichte einnimmt. Immerhin wird nur berichtet, dass der Architriklinos das Getränk kostet und seine herausragende Qualität bestätigt, nicht jedoch, dass dieses Getränk auch tatsächlich von den versammelten Hochzeitsgästen getrunken wird. Dies scheint, verglichen mit anderen Geschenkwundern, ein Mangel zu sein. So wird für das Geschenkwunder der Brotvermehrung (Joh 6,1-15 und par.) nicht nur berichtet, wie viele gesättigt werden, sondern auch noch, wie viel übrig bleibt. Auf dem Hintergrund dieser Beobachtung muss die Frage aufgeworfen werden, ob es nicht korrekter wäre, im Fall des Wunders bei der Hochzeit zu Kana von einem „Wandlungswunder" zu sprechen, da ja im eigentlichen Sinn nur die Wandlung, nicht jedoch der Genuss des gewandelten Getränks berichtet wird. ${ }^{142}$ „Zudem ist in der Kana-Perikope gar nicht die Hochzeits-, sondern einzig die Weinthematik zentral. ${ }^{143}$ Von diesem Befund her wird dann auch fraglich, ob weiterhin die Hochzeitals das zentrale theologische Motiv verstanden werden kann. ${ }^{144}$ Schließlich bemerkt Siegfried Bergler zurecht: „Letztlich ist diese Erzählung auch ohne den Hochzeitskasus zu verstehen. Das Wunder könnte sich genauso bei einem Symposion zugetragen haben. ${ }^{" 145}$

Die offensichtliche Fokussierung der Wundererzählung auf die Thematik der Wandlung, legt nun doch den Schluss nahe, dass Jesus hier als wirkmächtiger Wundertäter beschrieben wird, auch wenn dies teilweise bereits a priori ausgeschlossen wird. ${ }^{146}$

Eine Möglichkeit ist selbstverständlich, die vorliegenden Überlegungen als Argument dafür zu verwenden, dass Martin Hengel zuzustimmen ist,

142) Durch die Erzählstruktur wird die Wandlung in besonderer Weise hervorgehoben; vgl. B. Olsson, Structure and Meaning in the Fourth Gospel: A Text-Linguistic Analysis ofJohn 2:1-11 and 4:1-42 (CB.NT 6; Lund: CWK Gleerup, 1974) 82.

143) Bergler, Kana in Galiläa, 105. Dagegen jedoch R.E. Brown, The Gospel according to John (i-xii) (AncB 29; Garden City NY: Doubleday, 1966) 103: „Scholarly interpretations to the contrary, John does not put primary emphasis on the replacing of the water for Jewish purifications, nor on the action of changing water to wine (which is not described in detail), nor even on the resultant wine."

144) So z.B. Zimmermann, Christologie der Bilder, 211: „Die Offenbarung der Herrlichkeit Jesu geschieht demnach nicht nur auf einer Hochzeit, sondern gewissermaßen als Hochzeit. [...] Wenn der Leser nach Joh ifin. ein Offenbarungsgeschehen erwartet, erhält er durch Joh 2,1 den Hinweis, dass sich durch eine Hochzeit die Offenbarung ereignet."

145) Bergler, Kana in Galiläa, 105.

146) Collins, „Cana,“ $80:$ „[...] the reader of the Gospels must be aware that Jesus' nature miracles are generally told so as to illustrate a truth more profound than the simple fact that the historical Jesus of Nazareth was a thaumaturge." 
wenn er schreibt: „Nachdem die alttestamentlich-jüdische Wundertradition zahlreiche Vermehrungs- und Verwandlungswunder kannte und auch die Wein-Symbolik im Judentum selbst bis hinein in den Tempelkult und die eschatologische Erwartung eine ganz große Rolle spielte, wird man eine Verwandlung von Wasser in Wein kaum irgendwie als Fremdkörper empfunden haben. Sie paßte zum Messias Israels so gut wie zum heidnischen Gott. ${ }^{147}$ Dies scheint jedoch etwas zu kurz zu greifen.

Gerade deswegen scheint es sinnvoll, die Verse 6 bis 9 als zusammengehörige Elemente zu lesen: Ohne dass Jesus sich den Krügen genähert hat, wurde Wasser, das in Krüge gefüllt worden war, in denen sich noch nie ein Tropfen Wein befunden hatte und in die auch nichts anderes mehr hätte hineingefüllt werden können-Wein wurde ja in der Antike mit Wasser gemischt, bevor er getrunken wurde-, zu Wein, der von den Dienern geschöpft ${ }^{148}$ und vom Architriklinos für gut befunden wurde. Und die grundsätzliche Bedeutung dieser vier Verse könnte man wohl darin sehen, dass durch dieses Wunder und die Art, wie es geschieht, vor allem zum Ausdruck gebracht wird, dass Jesus das wirken kann, was auch Magier und Götter der Antike vermögen, ${ }^{149}$ dass es aber eben um ein Wunder und nicht um einen Trick geht. ${ }^{150}$ Derartige Wunder, die häufig Speisen betrafen, sind in der Antike bekannt. ${ }^{151}$ Auch scheinen jüdische Magier einen besonders guten Ruf gehabt zu haben. ${ }^{152}$ Ferner galt Jesus in rabbinischen Texten teilweise als Magier. ${ }^{153}$ Somit setzt diese Perikope selbstverständlich die

147) Hengel, „Der ,dionysische“ Messias,“ 6 oo.

148) Vgl. Zumstein, „Die Bibel als literarisches Kunstwerk,“ 76.

149) Entschieden dagegen jedoch Ashton, Understanding the Fourth Gospel, 175: „This in itself rules out of court the theory that the key to the story's meaning lies in the prodigious, that Jesus' primary role is that of thaumaturge, and that the content of the disciples' faith is their justified conviction in his power to work miracles."

150) Vgl. M. Becker, Wunder und Wundertäter im frührabbinischen Judentum: Studien zum Phänomen und seiner Überlieferung im Horizont von Magie und Dämonismus (WUNT 2/144; Tübingen: Mohr Siebeck, 2002) 139: „Dem Verbot der ,Zauberei‘ wird die Kategorie der ,Augentäuschung" hinzugefügt [...]."

151) Vgl. Hengel, „Der ,dionysische“ Messias,“ 593.

152) W. Kinzig, „,Non-Separation: Closeness and Co-Operation between Jews and Christians in the Fourth Century,“ VigChr 45 (1991) 27-53, hier 40: „At that time medicine and magic could not really be distinguished. In popular piety, however, magic played a major role. Jewish magic had the reputation of being particularly effective."

153) D. Boyarin, Dying for God: Martyrdom and the Making of Christianity and Judaism (Stanford: Stanford University Press, 1999) 38: „As we have seen, early rabbinic texts repeatedly refer to Jesus as a magician." 
biblischen Berichte von Wandlungswundern bei Flüssigkeiten wie auch bei Feststoffen und Farben voraus. ${ }^{154}$ Ferner darf man intertextuelle Bezüge zur Apostelgeschichte vermuten. ${ }^{155}$ Während in der Perikope von der Hochzeit zu Kana die Wirkmacht des Wundertäters erzählt wird, der keiner Täuschungsmanöver bedarf, wird bei dem Bericht vom Versuch des Magiers Simon, die Wunderkräfte der Apostel käuflich zu erwerben, vorausgesetzt, dass Wunder für den antiken Menschen sogar eine Einkommensquelle darstellen konnten. ${ }^{156}$ Letztlich spricht auch die bereits frühchristlich belegte Darstellung Christi mit dem Zauberstab (virga thaumaturga) für die hier vorgeschlagene Deutung. ${ }^{157}$ Doch, auch andere Stellen des Johannesevangeliums setzen voraus, dass durch Menschen gewirkte Wunder grundsätzlich möglich sind, dass jedoch wirkliche Wunder diesen Menschen in eine besondere Beziehung zu Gott setzen. ${ }^{158}$

Die Annahme, dass der Verfasser durch eine Bezugnahme auf die Wandlungswunder zu Beginn der Tätigkeit des Moses ${ }^{159}$ die Brücke sowohl zu den biblischen Vorbildern wie auch zu den in seinem hellenistischen Umfeld bekannten Wandlungswundern schlägt, ${ }^{160}$ berücksichtigt die vom

154) Dagegen jedoch Gnilka, Johannesevangelium, 23: „Im Gegensatz zum Heilungswunder wird das Geschenkwunder nicht beschrieben. Therapeuten gab es auch anderswo, Geschenkwunder entsprachen nicht dem Erfahrungshorizont."

155) Zur Konstitution von Intertextualität durch thematische Bezüge vgl. Gillmayr-Bucher, Intertextuality, 20.

156) Vgl. Apg 8:4-25.

157) Vgl. J.G. Deckers, Die frühchristliche und byzantinische Kunst (München: C.H. Beck, 2007) 47: „Um seine göttliche Fähigkeit anzudeuten, Wunder zu wirken, wird ihm zuweilen ein Zauberstab (Virga thaumaturga) in die Hand gegeben. " Siehe auch D. Knipp, ,Christus Medicus' in der frühchristlichen Sarkophagskulptur: Ikonographische Studien der Sepulkralkunst des späten vierten Jahrhunderts (SVigChr 37; Leiden: Brill, 1998) 15: „Tatsache ist, daß Christus mit der virga ausschließlich Tote erweckt (Lazarus, Jüngling von Naim) und Speisungswunder vollbringt, also nichts Lebendes berührt."

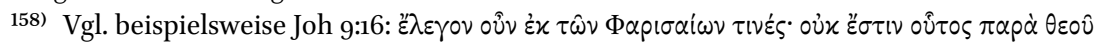

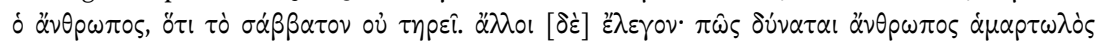

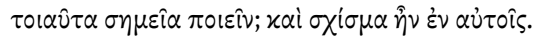

159) Selbstverständlich bestehen in der verwendeten Terminologie auch semantische Bezüge; vgl. grundsätzlich J. Lierman, „The Mosaic Pattern of John's Christology, “ in Challenging Perspectives on the Gospel of John (hrsg. J. Lierman; WUNT 2/219; Tübingen: Mohr Siebeck, 2006) 210-234; siehe auch H. Thyen, „Liegt dem Johannesevangelium eine SemeiaQuelle zugrunde?," in Studien zum Corpus Iohanneum (hrsg. H. Thyen; WUNT 214; Tübingen:

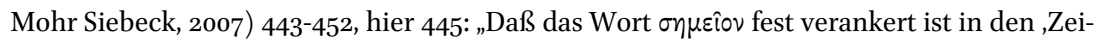
chen', die Mose in Ägypten getan hat, sieht Nicol wohl ganz richtig."

160) Vgl. hierzu P. Phillips, „Biblical Studies and Intertextuality: Should the Work of Genette and Eco Broaden our Horizons?, "in The Intertextuality of the Epistles: Explorations of Theory 
Verfasser rezipierten jüdischen Traditionen ohne Missachtung des antiken Umfelds. ${ }^{161}$ Und gerade am Anfang der Zeichen, die Moses gewirkt hat, findet sich nicht nur ein einziges, sondern eine Reihe von Wundern, die Stoffe bzw. Gegenstände verändern. ${ }^{162}$ So verwandelt Moses zweimal den Stab in eine Schlange - und zwar das erste Mal zum eigenen Erstaunen ${ }^{163}$ und das zweite Mal in Auseinandersetzung mit den Magiern des Pharao, wobei die Überbietung der Magier dadurch zum Ausdruck kommt, dass die Schlangen der Magier des Pharao von der Schlange, die Moses aus Aarons Stab gemacht hatte, gefressen werden. ${ }^{164}$ Auch die Hand des Mose wird weiß wie von Aussatz und danach wieder rein ${ }^{165}$ und die Verwandlung von Wasser in Blut ${ }^{166}$ wird sowohl von Moses wie von den Magiern des Pharao bewirkt. ${ }^{167}$ Da nun eine direkte Konkurrenzsituation mit anderen Wundertätern nicht Teil der vorliegenden Erzählung des Johannesevangeliums ist, wird dies, so scheint es, durch den Verfasser des Evangeliums indirekt thematisiert, indem die Möglichkeit einer Täuschung ausgeschlossen und die Tatsächlichkeit des Wunders durch die detaillierte Beschreibung des Wunders bezeugt wird. Dies dürfte auch dem Adressatenkreis der neutestamentlichen Schriften gerecht werden. ${ }^{168}$ Dionysische Wunder

and Practice (hrsg. T.L. Brodie, D.R. MacDonald und S.E. Porter; New Testament Monographs 16; Sheffield: Sheffield Phoenix Press, 2006) 35-45, hier 42: „Intertextuality actually signals a text's integration within a wider cultural setting, within a specific cultural identity.“

161) Dies ist zu betonen, da gerade exegetische Werke, welche die Verwurzelung des Johannesevangeliums im Judentum betonen, auf das hellenistische Umfeld zu wenig achten; vgl. hierzu Schnelle, „Literatur zum Johannesevangelium, “ 291: „Eine traditions- und religionsgeschichtliche Fehleinschätzung, denn das Johannesevangelium ist gerade mit seinen religionsphilosophischen Aussagen (vgl. Joh 1,1-4.14.18; 4,24; 10,30; 18,38; ferner 1 Joh 1,5; 4,8.16) Teil des Hellenismus!"

162) $\mathrm{Zu}$ den Parallelen bezüglich des Wandlungsmotivs zwischen Moses und Jesus vgl. auch M. Labahn, Jesus als Lebensspender: Untersuchungen zu einer Geschichte der johanneischen Tradition anhand ihrer Wundergeschichten (BZNW 98; Berlin/New York: De Gruyter, 1999) 147 .

163) $\operatorname{Ex} 4: 1-5$.

164) Ex 7:8-12.

165) Ex 4:6-7.

166) Siehe auch Ex 4:9.

167) Ex 7:14-25.

168) Vgl. G. Theissen, Die Entstehung des Neuen Testaments als literaturgeschichtliches Problem. Vorgetragen am 27.11.2004 (Heidelberg: Universitätsverlag Winter, 2007) 159: „Die neutestamentlichen Autoren mochten zu literaturfähigen gebildeten Kreisen gehören, die Adressaten der urchristlichen Schriften gehörten sicher in ihrer Mehrzahl zu literaturfernen Schichten." 
wären bei dieser Interpretation selbstverständlich im Sinne einer Anspielung ${ }^{169}$ Teil des größeren kulturellen Kontextes, ${ }^{170}$ jedoch nicht spezifisch oder gar ausschließlich durch diese Erzählung intendiert, da nicht nur dieser Gott Flüssigkeiten verwandelte, sondern selbst Magier, die Menschen sind, dazu in der Lage waren.

So verstanden, bildet dieses Wunder den Beginn des Lernprozesses der Jünger, an dessen Ende die Erfahrung der Auferstehung steht. Gerade weil sie auf ihrem Weg Jesus als einen Wundertäter kennen lernen, der zuerst Dinge vollbringt, die auch andere Thaumaturgen in der Antike vollbrachten, ist hier ein Lernprozess gegeben, der die Jünger als zutiefst in antiken Vorstellungen verwurzelt erweist. Und tatsächlich scheinen die Jünger im Evangelium das, was sie kennen, für möglich zu halten. Ein Beispiel hierfür wäre das johanneische Missverständnis bei der Auferweckung des Lazarus. ${ }^{171}$ In diesem Sinn ist eine intertextuelle Lektüre, die zu rasch die Symbolik der Hochzeit thematisiert, problematisch für das Verstehen der ursprünglichen Bedeutung des Textes. ${ }^{172}$ Offenbleiben muss im Zusammenhang dieser Überlegungen, was diese Fokussierung des Wunderberichts auf das Wandlungsmotiv im Zusammenhang des Johannesevangeliums bedeuten könnte, da dies weit über die Frage der Bedeutung papyrologisch-archäologischer Quellen für die Exegese der Kana-Perikope hinausführt.

169) Vgl. J. Paulien, „Elusive Allusions in the Apocalypse: Two Decades of Research into John's Use of the Old Testament," in The Intertextuality of the Epistles: Explorations of Theory and Practice (hrsg. T.L. Brodie, D.R. MacDonald und S.E. Porter; New Testament Monographs 16; Sheffield: Sheffield Phoenix Press, 2006) 61-68, hier 63: „The assessment of an ancient author's intention with regard to allusions will always be an exercise in probability.“ 170) Vgl. Becker, Wunder und Wundertäter, 139: „Zudem besteht trotz aller Kritik eine gemeinsame Prämisse in der Überzeugung, daß,Magie‘ existiert und wirkt. Diese Sicht stellt eine wichtige Voraussetzung für das Verständnis von,Wundern` und, Wundertätern' dar, da sie die Rabbinen zur Klärung der Verwechslungsmöglichkeit von ,Wunder‘ und „Magie‘ nötigte."

171) Vgl. H. Förster, „Johannes 11:11-14—ein typisches johanneisches Missverständnis?,“ NT 53 (2011) 338-357.

172) Dagegen Nicklas, „Biblische Texte,“ 254: „Für ein Verstehen des Textes in seinem biblischen Kontext sind selbst Einspielungen erlaubt, die dem Autor bzw. den Autoren des Johannesevangeliums eventuell nicht zugänglich waren." 